\title{
HIV/AIDS dynamics among pregnant women in Zanzibar
}

\author{
Latifa S. Ufuzo ${ }^{1,2}$, and Jean M. Tchuenche ${ }^{2}$ \\ ${ }^{* 1}$ Karume Institute of Technology, Mbweni, Zanzibar, Tanzania. \\ Tel: +255-77-746-7164, E-mail: latifau@yahoo.com \\ ${ }^{2}$ Mathematics Department, University of Dar es Salaam, Box 35062, Tanzania.
}

doi:10.5618/bio.2011.v1.n1.7 || Received: 12-12-2011, Accepted: 17-1-2012, Available online: 28-1-2012

\begin{abstract}
The 2003/04 Tanzania HIV indicator survey points that women are more likely to carry the highest burden than men, while at the same time, HIV prevalence among pregnant women in Zanzibar increased from $0.3 \%$ to $0.6 \%$ between 1980 and 1997 . A classical epidemiological framework via a determinestic mathematical model is provided. Sensitivity analysis on the key parameters driving the disease is carried out to determine their relative importance to initial disease transmission and prevalence. It turns out that the most sensitive parameter is the treatment rate.
\end{abstract}

Keywords: HIV/AIDS; ARV; Reproduction number; Sensitivity analysis.

\section{Introduction}

Despite remaining the leading infectious disease challenge in global health, the world has begun to reverse the spread of HIV on the cusp of the fourth decade of the HIV/AIDS epidemic, but the gains are still fragile. For the estimated 33.3 million people living with HIV after nearly 30 years into a very complex epidemic, about $70 \%$ are in sub-Saharan Africa [1]. A brief history of the disease in Tanzania provides the context of this study.

The first case of HIV/AIDS was reported in 1983 in the United Republic of Tanzania mainland. By 1985, an estimate of the number of people living with HIV/AIDS was about 140,000 (1.3\% prevalence) and by 1990 that estimate rose to 900,000 ( $7.2 \%$ prevalence). The surveillance report on HIV/AIDS and sexually transmitted infections for 2003 published by the National AIDS Programme estimated that more than 1.8 million people aged 0-59 years old were living with HIV/AIDS. Women are inordinately affected by the disease, especially pregnant women. The 2003-04 Tanzania HIV indicator survey showed an overall prevalence rate of
7.2\% (about 1 million people) among those aged 15-49 years old in the mainland. It indicated that women (7.7\%) are more likely to carry the highest burden than men (6.3\%). Mbeya and Iringa have HIV prevalence over $14 \%$, whereas other regions have about $2 \%$ prevalence. HIV in Zanzibar was first diagnosed in 1986 at Mnazi Mmoja hospital (Zanzibar town). The disease prevalence rate is estimated to be less than $1 \%$. However, with the expansion of substance abuse and other risky sexual behavior, the trend of HIV infection in Zanzibar is on the rise. The total number of people diagnosed in the Island increased from 3 in 1986 to an estimated 6000 in 2004 , and the prevalence rate among pregnant women also rose from $0.3 \%$ to $0.6 \%$ from 1980 to 1997 . This prevalence is higher in the urban areas $(0.9 \%)$ compared to rural areas $(0.5 \%)$; and it is higher in females $(0.9 \%)$ than in males $(0.2 \%)$. Statistics from 2002 showed that about $66.6 \%$ of people in Zanzibar live in rural areas, and $33.4 \%$ are urban dwellers [2]. The prevalence rate from 2005 to 2008 still fluctuates between $0.6 \%$ and $0.87 \%$. However, women are five times more likely to be HIV infected than their male counterparts in Zanzibar. Gender differences in HIV infections have been attributed to one of several reasons, which include the biological fact that HIV is easily transmitted from men-to-women. Furthermore, sexual activity tends to start earlier for women.

There are a number of strategies suggested to help reducing/eliminating the burden of HIV in a community [3], but no previous study focused on the effect of ARV therapy and the disease drivers in pregnant women in Zanzibar. It is therefore against this background that this study is carried out. Partial data collected from May 2007 to December 2008 in two representative ante-natal clinics show yearly trend of children that use Neverapine (started in 2007) immediately after birth (Figure 1). It is important to note that these children are born to infected mothers who themselves were on treatment. In these two health facilities, all children were tested prior to the drug regimen administration. 
Many of theses children were sero-negative while a few test results were undetermined (we only report determinate results). The primary goal is to theoretically determine which of the model parameters drives the epidemic in the presence/absence of antiretrovirals (ARVs). To this effect, a focus is placed on quantifying the effect of the disease drivers through a sensitivity analysis.

\section{Materials and Methods}

2.1 Dynamic framework. The one-sex structured progression model classifies the total women population $N_{w}$ into various subclasses according to their disease status, namely: susceptible women $W_{s}$, pregnant women with no HIV/AIDS $W_{p}$, infected women $W_{H}$, pregnant women with HIV $W_{H P}$, women with AIDS $W_{A}$, and women on ARVs $\mathrm{W}_{\mathrm{T}}$. Note that women with clinical AIDS are unlikely to get pregnant since they are or might be too sick to engage in sexual activities. The outflow from the population of pregnant women is $\phi$, a proportion $p$ of which move to the treatment class and the complementary proportion $1-p$ move directly to the AIDS class (since not everyone in need of treatment received it). Susceptible women acquire HIV infection at the rate $\lambda_{1}$ (effective contact rate).

2.2 Parameters estimation. Although much is known about the complex dynamics of HIV, there are still areas to explore in terms of what maintains the disease in the population. Since most field and lab data involve human subjects and therefore difficult to collect, mathematical models can fill in gaps, but they need as inputs the values of certain key demographic and epidemiological quantities which parameterize the models [4]. We survey the literature at our disposal and the partial data collected from two health care facilities in the Island of Zanzibar to determine the relative importance of the different factors that drive initial disease transmission and prevalence. Confidence in these conclusions is limited by a severe paucity of data underlying associated parameter estimates [4], but the result is informative enough and the approach developed herein can be adapted to the study of other sexually transmitted diseases.

The constant recruitment rate $\pi$ is obtained by dividing the total population of women who are most at risk between the ages of 15 and 35 in Zanzibar by the difference in age [5]. Thus, $\pi=79847 / 20=3992$. The average life expectancy is only 42 years for men and 44 years for women [5], therefore the average female mortality (natural death) rate $\mu_{1}$ for the given population is $1 / 44=0.02$, and the death rate due to disease $\mu_{2}$, is determined by following the methods used in [4]. The (number of pregnant women diagnosed with HIV) $\times$ (median survival time) $=$ (woman-months), where the total number of pregnant women for 4 years was about 11,747, the number of women diagnosed with HIV was 910, thus making an average of 227.51 pregnant women and the median survival time after diagnosis with HIV is about 7.8 years. The number of disease-induced deaths is 48 . Therefore, $\mu_{2}=$ death/(womanmonths $)=48 / 227.5 \times 7.8)=0.027 \mathrm{yr}^{-1}$.

Treatment coverage of infected individuals under ARVs is $\delta=0.951$. It is assumed that between $51-90 \%$ of infected women has access to ARVs treatment. $\phi$ is the average rate of leaving the infected class. We use the children 4 years data to estimate this parameter. $\phi=$ new infants/years $=283 / 4=70.75$ per 100 per year. Thus, $\phi=0.71$. This estimated value agrees with the value obtained in [6]. AIDS progression rate from the treated class is assumed to be 0.05 . The remaining parameter values are either pulled from the literature or assumed for the purpose of illustration and appear in Table 1.

\section{Results}

3.1 Sub-model without treatment. The basic reproduction number (i.e. the number of secondary infections generated by a single infected woman introduced into a completely naive/ susceptivle population [7]) is

$$
R_{0}=\frac{\lambda_{1}}{\mu_{1}+p \phi}\left(1+\frac{p \phi}{\mu_{1}+\mu_{2}}\right)
$$

3.2 Sub-model with treatment. The effective reproduction number (which measures the average number of new infections generated by a single infected person in a completely susceptible community in which treatment if available [7] is

$$
R_{0}=\frac{\lambda_{1} r_{1}}{\left(\mu_{1}+\mu_{2}\right)\left(r_{2}+\gamma+\delta+\mu_{1}\right)} \cdot \frac{\lambda_{2}\left(r_{3}+\varepsilon\right)}{\mu_{1} r_{3}+\left(p \phi+\mu_{1}\right)\left(\varepsilon+\mu_{1}\right)}
$$

2.3 Sensitivity analysis: The relative importance of model parameters to disease transmission is investigated using the normalized forward sensitivity index which is basically a deterministic one way sensitivity analysis in [8]. In determining how best to reduce human mortality and morbidity due to HIV/AIDS in pregnant women, it is necessary to know the relative importance of the different factors responsible for its transmission. The initial disease transmission is directly related to the reproduction number $R_{0}$, while the disease prevalence is 
directly related to the endemic equilibrium [8]. The parameter values in Table 1 are used to calculate the sensitivity indices (which measure the relative change in a state variable when a parameter changes). Note that the particular values of the sensitivity indices of the two reproduction numbers to different parameters depend on the parameter values chosen and on the assumptions upon which the model is based. The sensitivity indices of the reproduction numbers are computed and summarized in Table 2. The sign and the absolute value of the sensitivity index provide two different types of information. The absolute value of the sensitivity index shows the sensitivity of the state variable to the variation of the parameter, a high absolute value signifies the largest effect in the variable to the variation. Furthermore, a negative sensitive index value for a parameter means the variable is a decreasing function of the parameter.

The most sensitive and significant parameter on initial disease threshold parameter $R_{0}$ is the effective contact rate rate $\lambda_{I}$. Increasing $\lambda_{I}$ by
$10 \%$ increases $R_{0}$ by $10 \%$. For $R_{T}$, increasing $p$ by $10 \%$ reduces the initial disease transmission $R_{T}$ by $3.16 \%$. Providing ARVs early to infected pregnant women will greatly contribute in reducing/eliminating mother-to-child transmission. We note finally that the (natural death) rate $\mu_{l}$ is very sensitive, but in general, we have less control over it. However, for the disease-induced death rate $\mu_{2}$, treatment has a great impact in reducing or delaying deaths.

3.3 Sensitivity indices of the endemic equilibrium. The endemic equilibrium is numerically computed using the baseline parameter values in Table 1, and is given by

$$
E^{*}=(60.89,1.32,3654.46,5987.81,103739.65,27366.60) .
$$

Its sensitivity indices (also computed using the method described in [8]) are summarized in Table 3. The most sensitive parameter for infected pregnant women in the treatment model $\left(W_{H P}^{*}\right)$ other than the death rate and the progression rate to AIDS from the treated class is the rate of seeking treatment, $p$. The intuitive explanation of the sensitivity indices of the endemic equilibrium point is that, the increases in human population demographic parameters $\pi, r_{1}$ and $\lambda_{2}$ affect the size of the endemic equilibrium size.

Table 1. Model parameter description and their values.

\begin{tabular}{|l|l|l|l|}
\hline Parameter & Description & Value & Source \\
\hline$\mu_{1}$ & Natural death rate & 0.02 & Estimated \\
\hline$\mu_{2}$ & AIDS induced death rate & 0.03 & Estimated \\
\hline$\pi$ & Recruitment rate & 3992 & Estimated \\
\hline$r_{1}$ & Pregnancy rate of susceptible women & 0.03 & {$[9]$} \\
\hline$r_{2}$ & Rate of pregnant women acquiring infection & 0.02 & Assumed \\
\hline$r_{3}$ & Pregnancy rate of infected women under treatment & 0.01 & {$[9]$} \\
\hline$\phi$ & Rate of leaving the infected class & 0.71 & {$[6]$} \\
\hline$p$ & Proportion of pregnant women seeking treatment & 0.6 & {$[9]$} \\
\hline$\delta$ & Treatment rate of HIV+ & 0.51 & {$[9]$} \\
\hline$\varepsilon$ & Progression to AIDS from the treated class & 0.05 & Assumed \\
\hline$\gamma$ & Rate of leaving the infected to the AIDS class & 0.75 & Assumed \\
\hline$w$ & Pregnancy rate of infected women & 0.4 & {$[9]$} \\
\hline$\lambda_{1}, \lambda_{2}$ & Effective contact rates & $0.009 ; 0.42$ & Assumed \\
\hline$\varphi_{1}, \eta_{1}, v, \eta_{2}$ & Modification parameters & $1.1 ; 0.001$ & {$[5]$} \\
\hline
\end{tabular}


Table 2. Sensitivity indices of the basic and treatment-induced reproduction numbers ( $R_{0}$ and $R_{T}$ ).

\begin{tabular}{|l|c|c|}
\cline { 2 - 3 } \multicolumn{1}{c|}{} & \multicolumn{2}{l|}{ Sensitivity indices } \\
\hline Paremeter & $R_{0}$ & $R_{T}$ \\
\hline$\lambda_{1}$ & 0.35 & \\
\hline$\lambda_{2}$ & & 0.24 \\
\hline$p$ & -0.34 & -0.32 \\
\hline$\mu_{1}$ & -0.27 & -0.014 \\
\hline$\mu_{2}$ & -0.21 & -0.2 \\
\hline$\varepsilon$ & & -0.24 \\
\hline$\delta$ & & -0.18 \\
\hline$\gamma$ & & -0.15 \\
\hline
\end{tabular}

Table 3. Sensitivity indices of the endemic equilibrium.

\begin{tabular}{|l|c|c|c|c|c|}
\hline & \multicolumn{5}{|c|}{ Sensitivity indices } \\
\hline Paremeter & $W_{P}^{*}$ & $W_{H}^{*}$ & $W_{H P}^{*}$ & $W_{A}^{*}$ & $W_{T}^{*}$ \\
\hline$\lambda_{1}$ & -0.015 & 0.025 & & -0.02 & \\
\hline$\lambda_{2}$ & & 0.015 & & & \\
\hline$p$ & & -0.024 & -0.124 & & 0.039 \\
\hline$\mu_{1}$ & -0.063 & & & -0.09 & \\
\hline$\mu_{2}$ & & 0.028 & 0.085 & & -0.032 \\
\hline$\omega$ & -0.093 & -0.035 & 0.018 & & \\
\hline$r_{1}$ & & & & -0.014 & -0.14 \\
\hline$r_{2}$ & & -0.013 & & & \\
\hline $\boldsymbol{\varepsilon}$ & & 0.023 & -0.06 & & 0.02 \\
\hline$\delta$ & & -0.059 & 0.03 & & \\
\hline$\gamma$ & & 0.01 & & & 0.03 \\
\hline
\end{tabular}

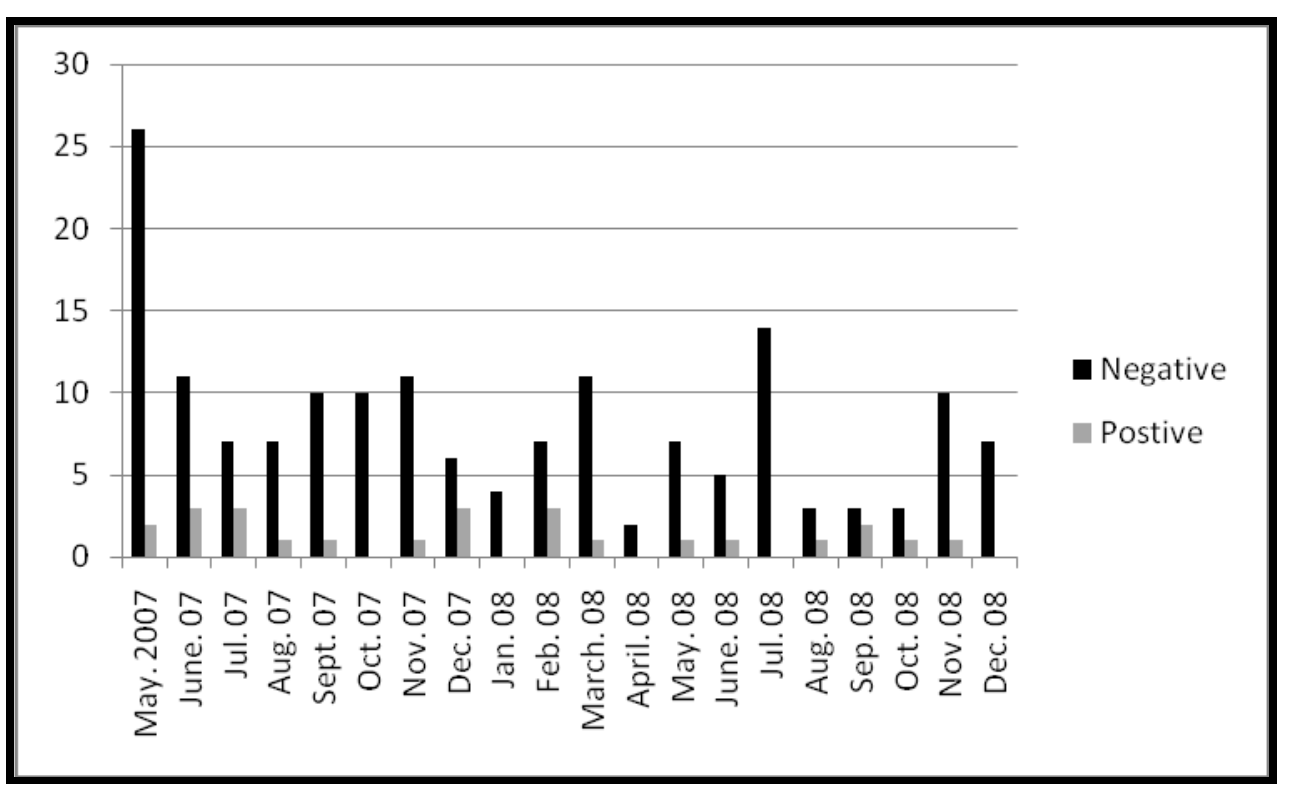

Figure 1. Yearly number of children that use Neverapine immediately after birth in two selected health care facilities in the Island of Zanzibar.

\section{Conclusion}

A deterministic compartment mathematical model is proposed. Two sub-models, namely: pregnant women without treatment and pregnant women with treatment are identified. The initial disease transmission for the various sub-models is computed via the next generation operator method. Sensitivity indices (which depend on the parameter values and the model assumptions) show that the most important parameters in relation to initial disease transmission for disease control are: the recruitment rate into susceptible population, and proportion of pregnant women who seek treatment along with their newborn. For the model with treatment, the most sensitive parameter for initial disease transmission is the rate of becoming pregnant, followed by the rate at which infected women under treatment become pregnant and the effective contact rate). Although intervention strategies cannot directly target the death rates 
and the progression to AIDS from the class of individuals under treatment, the treatment of infected/AIDS individuals including children born to these women has a positive population-level impact. To effectively guide public policy and public health decision making, the model and parameter values would need to be tested against data from antenatal clinics field sites. With some modifications and specific refinements, the proposed model can be used elsewhere to assess the impact of ARVs on infected pregnant women in meeting their reproductive health needs. For convenience, several assumptions are made; therefore our results are based on the formulation of the model. There are some limitations to this study, for instance, vertical transmission is not explicitly accounted for. The analysis provides rough sensitivity estimates based primarily on model parameter choice. A more realistic perspective can be achieved through the use of a two-sex model explicitly accounting for vertical transmission.

Nevertheless, our model captures the average characteristics of the dynamics of HIV/AIDS among pregnant women. Providing an explicit contact-tracing structure, behavioral change and accounting for concurrent partnership are also viable. However, the main goal of this work was to provide a theoretical framework where the dynamics of HIV/AIDS among pregnant women can be addressed. Disease drivers are identified and their potential impact on the disease dynamics is provided via a sensitivity analysis. Some critical trends are identified, and it is our hope that the theoretical results obtained from this study will stimulate further interest.

\section{Summary}

For the estimated 33.3 million people living with HIV after nearly 30 years into a very complex epidemic, about $70 \%$ are in sub-Saharan Africa. Women are inordinately affected by the disease, especially pregnant women. The 2003-04 Tanzania HIV indicator survey showed an overall prevalence rate of $7.2 \%$ among those aged 15-49 years old in the mainland. It indicated that women $(7.7 \%)$ are more likely to carry the highest burden than men $(6.3 \%)$. The disease prevalence is Zanzibar is estimated to be less than $1 \%$. However, with the expansion of substance abuse and other risky sexual behaviors, the trend of HIV infection in Zanzibar is on the increase. The total number of people diagnosed in the Island increased from 3 in 1986 to an estimated 6000 in 2004, and the prevalence rate among pregnant women also rose from $0.3 \%$ to $0.6 \%$ from 1980 to 1997 . Consequently, determining the driving factors of the disease is important.

A classical epidemiological framework via a determinestic mathematical model is provided. Sensitivity analysis on the key parameters driving the disease is carried out to determine their relative importance to initial disease transmission and prevalence. It turns out that the most sensitive parameter is the treatment rate. This result underscores the need to scale up treatment especially in hard hit settings.

\section{Acknowledgement}

LAU acknowledges with thanks the Karume Institute of Technology Zanzibar for study leave. D.M. Dahoma and H. Suleiman both of the Zanzibar AIDS Commission help in data collection and fruitful discussions.

\section{References}

[1] UNAIDS Global Report: UNAIDS/10.11E | JC1958E, 2010.

[2] MLYWCD, A report of assessment of HIV preventive services for young people in Zanzibar. 2008.

[3] Mukandavire Z.; Tchuenche, J.M.; Chiyaka C.; Musuka G. Advances in Disease Epidemiology, p. 221-241, Nova Science Publishers, NY, Inc, 2009.

[4] Kribs-Zaleta, C. PLoS Negl Trop Dis. 2010, 4(4) e656. http://dx.doi.org/10.1371/journal.pntd.0000656

[5] Pedro, S.A.; Tchuenche, J.M. J. Theor. Biol, 2010. 267(4), 471-485. http://dx.doi.org/10.1016/j.jtbi.2010.09.019

[6] Simwa, R.O.; Pokhariyal, G..P. Appl. Math. Comput., 2003, 146(1), 93-104. http://dx.doi.org/10.1016/S0096-3003(02)00528-3

[7] van den Driessche, P.; Watmough, J. Math Biosci, 2002, 180, 29-48. http://dx.doi.org/10.1016/S0025-5564(02)00108-6

[8] Chitnis, N., Hyman, J.M.; Cushing, J.M. Bull. Math. Biol., 2008, 70(5): p. 1272-96. http://dx.doi.org/10.1007/s11538-008-9299-0

[9] ZACP Report, http://www.who.int/hiv/HIVCP_TZA.pdf, 2008. 
The model equations are given by the following systems of differential equations:

$$
\begin{aligned}
& \frac{d W_{S}}{d t}=\pi-\lambda_{1} \omega W_{S}-\left(r_{1}+\mu_{1}\right) W_{S} \\
& \frac{d W_{P}}{d t}=r_{1} W_{S}-\lambda_{2} W_{P} \\
& \frac{d W_{H}}{d t}=(1-\omega) \lambda_{1} W_{S}-\left(r_{2}+\mu_{1}+\gamma+\delta\right) W_{H} \\
& \frac{d W_{H P}}{d t}=r_{2} W_{H}+\lambda_{2} W_{P}+r_{3} W_{T}+\omega \lambda_{1} W_{S}-\left(\mu_{1}+p \phi\right) W_{H P} \\
& \frac{d W_{A}}{d t}=(1-p) \phi W_{H P}+\gamma W_{H}+\varepsilon W_{T}-\left(\mu_{1}+\mu_{2}\right) W_{A} \\
& \frac{d W_{T}}{d t}=p \phi W_{H P}-\varepsilon W_{T}+\delta W_{H}-\left(\mu_{1}+r_{3}\right) W_{T}
\end{aligned}
$$

